\title{
The Revival of Kaon Flavour Physics
}

\author{
Andrzej J. Buras ${ }^{1,2, \star}$ \\ ${ }^{1}$ TUM Institute for Advanced Study, Lichtenbergstr. 2a, D-85748 Garching, Germany \\ ${ }^{2}$ Physik Department, Technische Universität München, James-Franck-Straße, D-85748 Garching, Germany
}

\begin{abstract}
After years of silence we should witness in the rest of this decade and in the next decade the revival of kaon flavour physics. This is not only because of the crucial measurements of the branching ratios for the rare decays $K^{+} \rightarrow \pi^{+} v \bar{v}$ and $K_{L} \rightarrow \pi^{0} v \bar{v}$ by NA62 and KOTO that being theoretically clean and very sensitive to new physics (NP) could hint for new phenomena even beyond the reach of the LHC without any significant theoretical uncertainties. Indeed simultaneously the advances in the calculations of perturbative and in particular non-perturbative QCD effects in $\varepsilon^{\prime} / \varepsilon, \varepsilon_{K}, \Delta M_{K}, K_{L} \rightarrow \mu^{+} \mu^{-}$ and $K_{L} \rightarrow \pi^{0} \ell^{+} \ell^{-}$will increase the role of these observables in searching for NP. In fact the hints for NP contributing to $\varepsilon^{\prime} / \varepsilon$ have been already signalled last year through improved estimates of hadronic matrix elements of QCD and electroweak penguin operators $Q_{6}$ and $Q_{8}$ by lattice QCD and large $N$ dual QCD approach. This talk summarizes in addition to this new flavour anomaly the present highlights of this field including some results from concrete NP scenarios.
\end{abstract}

\section{Introduction}

In two recent reports $[1,2]$ I have stressed the importance of kaon flavour physics in the search for new physics (NP) expressing my excitement in view of the revival of this field which played such an important role in the construction of the present theory of elementary particle physics represented by the Standard Model (SM) of strong and electroweak interactions. The goal of this writing is to list the most important advances in this field which have been made in the last two years since the last workshop of this series. Many of the topics listed below have already been discussed in [1-3] but I will present them from a different perspective and will add new ones. Moreover, I think it is useful to have a list of most important findings which can be looked up faster than in the longer expositions in [1-3]. Further details, in particular numerous references, can be found there.

\section{Important Messages}

\section{$2.1 \varepsilon^{\prime} / \varepsilon$}

Presently in kaon flavour physics the most exciting appears to be the anomaly in $\varepsilon^{\prime} / \varepsilon$ and we will look at it first. The present status of $\varepsilon^{\prime} / \varepsilon$ in the SM can be summarized as follows. The RBC-UKQCD

^e-mail: aburas@ph.tum.de 
lattice collaboration calculating hadronic matrix elements of all operators but not including isospin breaking effects finds $[4,5]$

$$
\left(\varepsilon^{\prime} / \varepsilon\right)_{\mathrm{SM}}=(1.38 \pm 6.90) \times 10^{-4}, \quad(\mathrm{RBC}-\mathrm{UKQCD}) .
$$

Using the hadronic matrix elements of QCD- and EW-penguin $(V-A) \otimes(V+A)$ operators from RBCUKQCD lattice collaboration but extracting the matrix elements of penguin $(V-A) \otimes(V-A)$ operators from the CP-conserving $K \rightarrow \pi \pi$ amplitudes and including isospin breaking effects one finds [6]

$$
\left(\varepsilon^{\prime} / \varepsilon\right)_{\mathrm{SM}}=(1.9 \pm 4.5) \times 10^{-4}, \quad(\mathrm{BGJJ}) .
$$

This result differs by $2.9 \sigma$ significance from the experimental world average from NA48 [7] and $\mathrm{KTeV}[8,9]$ collaborations,

$$
\left(\varepsilon^{\prime} / \varepsilon\right)_{\exp }=(16.6 \pm 2.3) \times 10^{-4},
$$

suggesting that models providing enhancement of $\varepsilon^{\prime} / \varepsilon$ are favoured. A new analysis in [10] confirms these findings

$$
\left(\varepsilon^{\prime} / \varepsilon\right)_{\mathrm{SM}}=(0.96 \pm 4.96) \times 10^{-4}, \quad(\mathrm{KNT}) .
$$

All these results are based on NLO calculations of the Wilson coefficients of the relevant operators that have been completed 23 years ago [11-16]. Partial NNLO calculations have been performed in [17-19]. Complete NNLO result from Maria Cerda-Sevilla, Martin Gorbahn, Sebastian Jäger and Ahmet Kokulu should be available soon.

While these results, based on the hadronic matrix elements from RBC-UKQCD lattice collaboration, suggest some evidence for the presence of NP in hadronic $K$ decays, the large uncertainties in the hadronic matrix elements in question do not yet preclude that eventually the SM will agree with data. In this context the upper bounds on the matrix elements of the dominant penguin operators from large $N$ dual QCD approach [20] are important as they give presently the strongest support to the anomaly in question, certainly stronger than present lattice results. To see this in explicit terms let us look at the parameters $B_{6}^{(1 / 2)}$ and $B_{8}^{(3 / 2)}$ that represent the relevant hadronic matrix elements of the QCD penguin operator $Q_{6}$ and the electroweak penguin operator $Q_{8}$, respectively.

In the strict large $N$ limit [21-23] one simply has

$$
B_{6}^{(1 / 2)}=B_{8}^{(3 / 2)}=1, \quad(\text { large N Limit }) .
$$

But RBC-UKQCD results [4, 5] imply [6, 24]

$$
B_{6}^{(1 / 2)}=0.57 \pm 0.19, \quad B_{8}^{(3 / 2)}=0.76 \pm 0.05, \quad(\text { RBC-UKQCD }),
$$

and this suppression of both parameters below unity, in particular of $B_{6}^{(1 / 2)}$, is the main origin of the strong suppression of $\varepsilon^{\prime} / \varepsilon$ within the SM below the data. Yet in view of the large error in $B_{6}^{(1 / 2)}$ one could be sceptical about claims made by me and my collaborators that there is NP in $\varepsilon^{\prime} / \varepsilon$. Future lattice results could in principle raise $B_{6}^{(1 / 2)}$ towards its large $N$ value and above $B_{8}^{(3 / 2)}$ bringing the SM result for $\varepsilon^{\prime} / \varepsilon$ close to its experimental value.

However, the analyses of $B_{6}^{(1 / 2)}$ and $B_{8}^{(3 / 2)}$ within the dual QCD approach in [20,25] show that such a situation is rather unlikely. Indeed, in this approach going beyond the strict large $N$ limit one can understand the suppression of $B_{6}^{(1 / 2)}$ and $B_{8}^{(3 / 2)}$ below the unity as the effect of the meson evolution from scales $\mu=O\left(m_{\pi}, m_{K}\right)$ at which (5) is valid to $\mu=O(1 \mathrm{GeV})$ at which Wilson coefficients of 
$Q_{6}$ and $Q_{8}$ are evaluated [20]. This evolution has to be matched to the usual perturbative quark evolution for scales higher than $1 \mathrm{GeV}$ and in fact the supressions in question and the property that $B_{6}^{(1 / 2)}$ is more strongly suppressed than $B_{8}^{(3 / 2)}$ are consistent with the perturbative evolution of these parameters above $\mu=O(1 \mathrm{GeV})$. Thus we are rather confident that [20]

$$
B_{6}^{(1 / 2)}<B_{8}^{(3 / 2)}<1 \quad \text { (dual QCD) }
$$

Explicit calculation in this approach gives $B_{8}^{(3 / 2)}\left(m_{c}\right)=0.80 \pm 0.10$. The result for $B_{6}^{(1 / 2)}$ is less precise but in agreement with (6). For further details, see [20].

It should be recalled that in the past values $B_{6}^{(1 / 2)}=B_{8}^{(3 / 2)}=1.0$ have been combined in phenomenological applications with the Wilson coefficients evaluated at scales $\mu=O(1 \mathrm{GeV})$. The discussion above shows that this is incorrect. The meson evolution from $\mu=O\left(m_{\pi}, m_{K}\right)$ to $\mu=O(1 \mathrm{GeV})$ has to be performed and this effect turns out to be stronger than the scale dependence of $B_{6}^{(1 / 2)}$ and $B_{8}^{(3 / 2)}$ in the perturbative regime, where it is very weak.

Additional support for the small value of $\varepsilon^{\prime} / \varepsilon$ in the SM comes from the recent reconsideration of the role of final state interactions (FSI) in $\varepsilon^{\prime} / \varepsilon$ [25]. Already long time ago the chiral perturbation theory practitioners put forward the idea that both the amplitude $\operatorname{Re} A_{0}$, governed by the current-current operator $Q_{2}-Q_{1}$ and the $Q_{6}$ contribution to the ratio $\varepsilon^{\prime} / \varepsilon$ could be enhanced significantly through FSI in a correlated manner [26-32] bringing the values of $\varepsilon^{\prime} / \varepsilon$ close to its experimental value. However, as shown recently in [25] FSI are likely to be important for the $\Delta I=1 / 2$ rule, in agreement with [26-32], but much less relevant for $\varepsilon^{\prime} / \varepsilon$. Even if the analysis in [25] is rather qualitative, it makes us belive that future more precise calculations will find $\varepsilon^{\prime} / \varepsilon$ well below its SM value. It should also be emphasized that the authors of [26-32] did not include the meson evolution of $B_{6}^{(1 / 2)}$ and $B_{8}^{(3 / 2)}$ in their analysis and already this effect would significantly lower their predictions for $\varepsilon^{\prime} / \varepsilon$.

It should finally be noted that even without lattice results, varying all input parameters, the bound in (7) implies the upper bound on $\varepsilon^{\prime} / \varepsilon$ in the SM

$$
\left(\varepsilon^{\prime} / \varepsilon\right)_{\mathrm{SM}}<(8.6 \pm 3.2) \times 10^{-4}, \quad(\mathrm{BG}) .
$$

On the other hand employing the lattice value for $B_{8}^{(3 / 2)}$ in $(6)$ and $B_{6}^{(1 / 2)}=B_{8}^{(3 / 2)}=0.76$, one obtains $(6.0 \pm 2.4) \times 10^{-4}$ instead of $(8)$, well below the data.

All these findings give strong motivation for searching for NP which could enhance $\varepsilon^{\prime} / \varepsilon$ above its $\mathrm{SM}$ value. We will summarize the present efforts in this direction below.

\subsection{Tensions between $\varepsilon_{K}$ and $\Delta M_{s, d}$ in the SM and CMFV Models}

In [33] we have pointed out a significant tension between $\varepsilon_{K}$ and $\Delta M_{s, d}$ within the SM and models with constrained MFV (CMFV) implied by new lattice QCD results from Fermilab Lattice and MILC Collaborations [34] on $B_{s, d}^{0}-\bar{B}_{s, d}^{0}$ hadronic matrix elements. Not everybody agrees on this tension as in allover fits this tension is not transparently seen. But plots in [33], in particular in Fig. 5 of that paper, show that there is a clear tension between $\varepsilon_{K}$ and $\Delta M_{s, d}$ in the SM and CMFV models. Our strategy was to ignore the present tree-level values of $\left|V_{c b}\right|$ and $\left|V_{u b}\right|$ in view of some discrepancies between their exclusive and inclusive determinations and to show that this tension persists independently of the values of CKM parameters. For smaller (exclusive) values of $\left|V_{c b}\right|$ one finds $\Delta M_{s, d}$ to agree well with the data, while $\varepsilon_{K}$ is roughly $25 \%$ below its experimental value. For $\left|V_{c b}\right|$ in the ballpark of inclusive determinations one finds $\varepsilon_{K}$ to agree with the data, while $\Delta M_{s, d}$ are then typically by $15 \%$ larger than their experimental values. These numbers are for the SM, in all other CMFV models the situation gets worse. 
But on the whole this tension is certainly not as large as is the case of the $\varepsilon^{\prime} / \varepsilon$ anomaly. We are looking forward to improved hadronic matrix elements in question from other lattice collaborations and to improved values of $\left|V_{c b}\right|$ and $\left|V_{u b}\right|$ which would tell us whether this tension persists and if this will turn out to be the case, whether there is a $\varepsilon_{K}$ anomaly and/or a $\Delta M_{s, d}$ anomaly.

\section{$2.3 K^{+} \rightarrow \pi^{+} v \bar{v}$ and $K_{L} \rightarrow \pi^{0} v \bar{v}$ in the SM}

These two rare decays allow to test the short distance scales far beyond the reach of the LHC. Even scales of $O(100) \mathrm{TeV}$ can be probed in this manner [35]. The present status of $K^{+} \rightarrow \pi^{+} v \bar{v}$ and $K_{L} \rightarrow \pi^{0} v \bar{v}$ within the SM has been presented in [24] with the result

$$
\begin{aligned}
\mathcal{B}\left(K^{+} \rightarrow \pi^{+} v \bar{v}\right) & =(8.4 \pm 1.0) \times 10^{-11}, \\
\mathcal{B}\left(K_{L} \rightarrow \pi^{0} v \bar{v}\right) & =(3.4 \pm 0.6) \times 10^{-11} .
\end{aligned}
$$

But the most important outcome of this paper are parametric expressions for the branching ratios of these two decays in terms of the CKM input and the correlations between $K^{+} \rightarrow \pi^{+} v \bar{v}$ and $B_{s} \rightarrow \mu^{+} \mu^{-}$ and between $K^{+} \rightarrow \pi^{+} v \bar{v}$ and $\varepsilon_{K}$ in the SM. These formulae should be useful for monitoring the numerical values for these branching ratios within the SM when the CKM input improves. On the other hand the results for both decays obtained in simplified models with flavour violating couplings of the SM $Z$ and of a heavy $Z^{\prime}$ can be found in [36]. A more general study of such models, performed in [37], will be discussed now.

\subsection{Strategy for $\varepsilon^{\prime} / \varepsilon$ and Lessons}

The present error on $\varepsilon^{\prime} / \varepsilon$ within the SM is still very large and it is rather inconvenient to carry it to NP models. Therefore in order to investigate the implications of $\varepsilon^{\prime} / \varepsilon$ anomaly on rare decays $K^{+} \rightarrow \pi^{+} v \bar{v}$ and $K_{L} \rightarrow \pi^{0} v \bar{v}$ in a systematic fashion a strategy has been proposed in [37]. While $\varepsilon^{\prime} / \varepsilon$ plays the dominant role in this strategy it was useful to assume that there is also a modest $\varepsilon_{K}$ anomaly.

Then $\varepsilon^{\prime} / \varepsilon$ and $\varepsilon_{K}$ in the presence of NP contributions are given by

$$
\frac{\varepsilon^{\prime}}{\varepsilon}=\left(\frac{\varepsilon^{\prime}}{\varepsilon}\right)^{\mathrm{SM}}+\left(\frac{\varepsilon^{\prime}}{\varepsilon}\right)^{\mathrm{NP}}, \quad \varepsilon_{K} \equiv e^{i \varphi_{\varepsilon}}\left[\varepsilon_{K}^{\mathrm{SM}}+\varepsilon_{K}^{\mathrm{NP}}\right]
$$

with NP contributions parametrized as follows:

$$
\left(\frac{\varepsilon^{\prime}}{\varepsilon}\right)^{\mathrm{NP}}=\kappa_{\varepsilon^{\prime}} \cdot 10^{-3}, \quad 0.5 \leq \kappa_{\varepsilon^{\prime}} \leq 1.5,
$$

and

$$
\varepsilon_{K}^{\mathrm{NP}}=\kappa_{\varepsilon} \cdot 10^{-3}, \quad 0.1 \leq \kappa_{\varepsilon} \leq 0.4 .
$$

The ranges for $\kappa_{\varepsilon^{\prime}}$ and $\kappa_{\varepsilon}$ indicate the required size of this contribution but can be kept as free parameters. They will be determined one day when the theory on $\varepsilon^{\prime} / \varepsilon$ and the CKM input improve.

In the simplest NP scenarios with tree-level $Z$ and $Z^{\prime}$ exchanges, the imaginary parts of flavourviolating $Z$ or $Z^{\prime}$ couplings to quarks are then determined as functions of $\kappa_{\varepsilon^{\prime}}$. As $\varepsilon_{K}$ is governed by the product of imaginary and real parts, invoking (13) allows then to determine the corresponding real parts as functions of $\kappa_{\varepsilon^{\prime}}$ and $\kappa_{\varepsilon}$.

Having fixed the flavour violating couplings of $Z$ or $Z^{\prime}$ in this manner, one can express NP contributions to the branching ratios for $K^{+} \rightarrow \pi^{+} v \bar{v}, K_{L} \rightarrow \pi^{0} v \bar{v}, K_{L} \rightarrow \mu^{+} \mu^{-}$and to $\Delta M_{K}$ in terms of $\kappa_{\varepsilon^{\prime}}$ 
and $\kappa_{\varepsilon}$. Explicit formulae can be found in [37]. In this manner one can directly study the impact of $\varepsilon^{\prime} / \varepsilon$ and $\varepsilon_{K}$ anomalies in $Z$ and $Z^{\prime}$ scenarios on these four observables. The pattern of flavour violation depends in a given NP scenario on the relative size of real and imaginary parts of the couplings involved and we will see this explicitly in the lessons below.

In [37] numerous plots for the ratios

$$
R_{+}^{v \bar{v}} \equiv \frac{\mathcal{B}\left(K^{+} \rightarrow \pi^{+} v \bar{v}\right)}{\mathcal{B}\left(K^{+} \rightarrow \pi^{+} v \bar{v}\right)_{\mathrm{SM}}}, \quad R_{0}^{v \bar{v}} \equiv \frac{\mathcal{B}\left(K_{L} \rightarrow \pi^{0} v \bar{v}\right)}{\mathcal{B}\left(K_{L} \rightarrow \pi^{0} v \bar{v}\right)_{\mathrm{SM}}}
$$

as functions of $\kappa_{\varepsilon^{\prime}}$ and $\kappa_{\varepsilon}$ within the models with tree-level $Z$ and $Z^{\prime}$ exchanges have been presented. In view of space limitations we will not repeat them here but instead we will list the most important lessons from this study defining flavour violating couplings $\Delta_{L, R}^{s d}(Z)$ by [38]

$$
i \mathcal{L}(Z)=i\left[\Delta_{L}^{s d}(Z)\left(\bar{s} \gamma^{\mu} P_{L} d\right)+\Delta_{R}^{s d}(Z)\left(\bar{s} \gamma^{\mu} P_{R} d\right)\right] Z_{\mu}, \quad P_{L, R}=\frac{1}{2}\left(1 \mp \gamma_{5}\right)
$$

with analogous definitions for $Z^{\prime}$ couplings. Moreover, we will use the abbreviations:

$$
\text { LHS } \equiv \text { left }- \text { handed scenario, } \quad \text { RHS } \equiv \text { right }- \text { handed scenario }
$$

for NP scenarios in which only left-handed $(\mathrm{LH})$ or right-handed $(\mathrm{RH})$ flavour-violating couplings are present.

Lesson 1: In the LHS, a given request for the enhancement of $\varepsilon^{\prime} / \varepsilon$ determines the coupling $\operatorname{Im} \Delta_{L}^{s d}(Z)$

Lesson 2: In LHS there is a direct unique implication of an enhanced $\varepsilon^{\prime} / \varepsilon$ on $K_{L} \rightarrow \pi^{0} v \bar{v}$ : suppression of $\mathcal{B}\left(K_{L} \rightarrow \pi^{0} v \bar{v}\right)$. This property is known from NP scenarios in which NP to $K_{L} \rightarrow \pi^{0} v \bar{v}$ and $\varepsilon^{\prime} / \varepsilon$ enters dominantly through the modification of $Z$-penguins.

Lesson 3: The imposition of the $K_{L} \rightarrow \mu^{+} \mu^{-}$constraint in LHS determines the range for $\operatorname{Re} \Delta_{L}^{s d}(Z)$ which with the already fixed $\operatorname{Im} \Delta_{L}^{s d}(Z)$ allows to calculate the shifts in $\varepsilon_{K}$ and $\Delta M_{K}$. These shifts turn out to be very small for $\varepsilon_{K}$ and negligible for $\Delta M_{K}$.

Lesson 4: With fixed $\operatorname{Im} \Delta_{L}^{s d}(Z)$ and the allowed range for $\operatorname{Re} \Delta_{L}^{s d}(Z)$, the range for $\mathcal{B}\left(K^{+} \rightarrow \pi^{+} v \bar{v}\right)$ can be obtained. Both an enhancement and a suppression of $\mathcal{B}\left(K^{+} \rightarrow \pi^{+} v \bar{v}\right)$ are possible. $\mathcal{B}\left(K^{+} \rightarrow\right.$ $\pi^{+} v \bar{v}$ ) can be enhanced by a factor of 2 at most.

Lesson 5: Analogous pattern is found in RHS, although the numerics is different. See Fig. 1 in [37]. In particular the suppression of $\mathcal{B}\left(K_{L} \rightarrow \pi^{0} v \bar{v}\right)$ for a given $\kappa_{\mathcal{E}^{\prime}}$ is smaller. Moreover, an enhancement of $\mathcal{B}\left(K^{+} \rightarrow \pi^{+} v \bar{v}\right)$ up to a factor of 5.7 is possible.

Lesson 6: In a general $Z$ scenario with $\mathrm{LH}$ and $\mathrm{RH}$ flavour-violating couplings the pattern of NP effects changes because of the appearance of LR operators dominating NP contributions to $\varepsilon_{K}$ and $\Delta M_{K}$. The main virtue of the general scenario is the possibility of enhancing simultaneously $\varepsilon^{\prime} / \varepsilon, \varepsilon_{K}$, $\mathcal{B}\left(K^{+} \rightarrow \pi^{+} v \bar{v}\right)$ and $\mathcal{B}\left(K_{L} \rightarrow \pi^{0} v \bar{v}\right)$ which is not possible in LHS and RHS. Thus the presence of both $\mathrm{LH}$ and RH flavour-violating currents is essential for obtaining simultaneously the enhancements in question. The correlations between $\varepsilon^{\prime} / \varepsilon$ and $K^{+} \rightarrow \pi^{+} v \bar{v}$ and $K_{L} \rightarrow \pi^{0} v \bar{v}$ depend sensitively on the ratio of real and imaginary parts of the flavour-violating couplings involved. But the main message from this analysis is that in the presence of both $\mathrm{LH}$ and RH flavour-violating couplings of $Z$ to quarks, large departures from SM predictions for $K^{+} \rightarrow \pi^{+} \nu \bar{v}$ and $K_{L} \rightarrow \pi^{0} v \bar{v}$ are possible and $\varepsilon^{\prime} / \varepsilon$ anomaly can be explained.

$Z^{\prime}$ models exhibit quite different pattern of NP effects in the $K$ meson system than the LH and RH $Z$ scenarios. In $Z$ scenarios only electroweak penguins (EWP) can contribute to $\varepsilon^{\prime} / \varepsilon$ in an important manner because of flavour dependent diagonal $Z$ coupling to quarks. But in $Z^{\prime}$ models the diagonal 
quark couplings can be flavour universal so that QCD penguin operators (QCDP) can dominate NP contributions to $\varepsilon^{\prime} / \varepsilon$. Interestingly, the pattern of NP in rare $K$ decays depends on whether NP in $\varepsilon^{\prime} / \varepsilon$ is dominated by QCDP or EWP operators. Moreover, the striking difference from $Z$ scenarios, known already from previous studies, is the increased importance of the constraints from $\Delta F=2$ observables.

The new finding in [37] is a large hierarchy between real and imaginary parts of the flavour violating couplings implied by anomalies in QCDP and EWP scenarios. In the case of QCDP imaginary parts dominate over the real ones, while in the case of EWP this hierarchy is opposite unless the $\varepsilon_{K}$ anomaly is absent. Because of these different patterns there are striking differences in the implications of the $\varepsilon^{\prime} / \varepsilon$ anomaly for the correlation between $K^{+} \rightarrow \pi^{+} v \bar{v}$ and $K_{L} \rightarrow \pi^{0} v \bar{v}$ in these two NP scenarios if significant NP contributions to $\varepsilon^{\prime} / \varepsilon$ are required. The plots in [37] and in particular analytic derivations presented there illustrate these differences in a spectacular manner. The main lessons are as follows.

Lesson 7: In the case of QCDP scenario the correlation between $\mathcal{B}\left(K_{L} \rightarrow \pi^{0} v \bar{v}\right)$ and $\mathcal{B}\left(K^{+} \rightarrow\right.$ $\left.\pi^{+} v \bar{v}\right)$ takes place along the branch parallel to the Grossman-Nir bound [39].

Lesson 8: In the EWP scenario this correlation between $\mathcal{B}\left(K_{L} \rightarrow \pi^{0} v \bar{v}\right)$ and $\mathcal{B}\left(K^{+} \rightarrow \pi^{+} v \bar{v}\right)$ proceeds away from this branch for diagonal quark couplings $O(1)$ if NP in $\varepsilon_{K}$ is present and it is very different from the one of the QCDP case. NP effects in rare $K$ decays turn out to be modest in this case unless the diagonal quark couplings are $O\left(10^{-2}\right)$ and then the requirement of shifting upwards $\varepsilon^{\prime} / \varepsilon$ implies large effects in $K^{+} \rightarrow \pi^{+} v \bar{v}$ and $K_{L} \rightarrow \pi^{0} v \bar{v}$ also in the EWP scenario.

Lesson 9: For fixed values of the neutrino and diagonal quark couplings in $\varepsilon^{\prime} / \varepsilon$ the predicted enhancements of $\mathcal{B}\left(K_{L} \rightarrow \pi^{0} v \bar{v}\right)$ and $\mathcal{B}\left(K^{+} \rightarrow \pi^{+} v \bar{v}\right)$ are much larger when NP in QCDP is required to remove the $\varepsilon^{\prime} / \varepsilon$ anomaly than it is the case of EWP.

Lesson 10: In QCDP scenario $\Delta M_{K}$ is suppressed and this effect increases with increasing $M_{Z^{\prime}}$ whereas in the EWP scenario $\Delta M_{K}$ is enhanced and this effect decreases with increasing $M_{Z^{\prime}}$ as long as real couplings dominate. Already on the basis of this property one could differentiate between these two scenarios when the SM prediction for $\Delta M_{K}$ improves.

\section{Results in specific NP models}

\subsection{Preliminaries}

While the $\varepsilon^{\prime} / \varepsilon$ anomaly identified in 2015 was shadowed until recently by the $750 \mathrm{GeV}$ resonance, the death of the latter will likely increase the interest in this new flavour anomaly. In particular the recent indications from the dual QCD approach that FSI are much less relevant for $\varepsilon^{\prime} / \varepsilon$ than previously expected and the upper bounds on $B_{6}^{(1 / 2)}$ and $B_{8}^{(3 / 2)}$ from this approach diminished significantly hopes that improved lattice calculations would bring the SM prediction for $\varepsilon^{\prime} / \varepsilon$ to agree with the experimental data, opening thereby an arena for important NP contributions to this ratio. The latest analyses of such contributions in the context of models with tree-level $Z$ and $Z^{\prime}$ exchanges like 331 models, Littlest Higgs model with T-parity can be found in [36, 37, 40-42]. The analyses in supersymmetric models can be found in [43-45]. In view of space limitations we will only briefly summarize the results in 331 models and the very recent results in models with vector-like quarks.

\subsection{Flavour News}

The 331 models are based on the gauge group $S U(3)_{C} \times S U(3)_{L} \times U(1)_{X}$ [46-50]. In these models new contributions to $\varepsilon^{\prime} / \varepsilon$ and other flavour observables are dominated by tree-level exchanges of a $Z^{\prime}$ with non-negligible contributions from tree-level $Z$ exchanges generated through the $Z-Z^{\prime}$ mixing. 
The size of these NP effects depends not only on $M_{Z^{\prime}}$ but in particular on a parameter $\beta$, which distinguishes between various 331 models, on fermion representations under the gauge group and a parameter $\tan \bar{\beta}$ present in the $Z-Z^{\prime}$ mixing. Extensive recent analyses in these models can be found in $[41,42,51-53]$. References to earlier analyses of flavour physics in 331 models can be found there and in $[54,55]$.

A detailed analysis of 331 models with different values of $\beta, \tan \bar{\beta}$ for two fermion representations $F_{1}$ and $F_{2}$, with the third SM quark generation belonging respectively to an antitriplet and a triplet under the $S U(3)_{L}$, has been presented in [53]: 24 models in total. Requiring that these models perform at least as well as the SM, as far as electroweak tests are concerned, seven models have been selected for a more detailed study of FCNC processes. Recent updated analyses of these seven models, that address the $\varepsilon^{\prime} / \varepsilon$ anomaly, have been presented in [41, 42] and we summarize the main results of these two papers putting the emphasis on the last analysis in [42] which could take into account new lattice QCD results from Fermilab Lattice and MILC Collaborations [34] on $B_{s, d}^{0}-\bar{B}_{s, d}^{0}$ hadronic matrix elements.

The new analyses in $[41,42]$ show that the impact of a required enhancement of $\varepsilon^{\prime} / \varepsilon$ on other flavour observables is significant. The one in [42] also shows that the results are rather sensitive to the value of $\left|V_{c b}\right|$ which has been illustrated there by choosing two values: $\left|V_{c b}\right|=0.040$ and $\left|V_{c b}\right|=0.042$.

The main findings of $[41,42]$ for $M_{Z^{\prime}}=3 \mathrm{TeV}$ are as follows:

- Among seven 331 models singled out through electroweak precision study only three (M8, M9, M16) can provide for both choices of $\left|V_{c b}\right|$, significant shift of $\varepsilon^{\prime} / \varepsilon$ but not larger than $6 \times 10^{-4}$, that is $\kappa_{\mathcal{E}^{\prime}} \leq 0.6$.

- The tensions between $\Delta M_{s, d}$ and $\varepsilon_{K}$, discussed previously can be removed in these models (M8, M9, M16) for both values of $\left|V_{c b}\right|$.

- Two of them (M8 and M9) can simultaneously suppress $B_{s} \rightarrow \mu^{+} \mu^{-}$by at most $10 \%$ and $20 \%$ for $\left|V_{c b}\right|=0.042$ and $\left|V_{c b}\right|=0.040$, respectively. This can still bring the theory within $1 \sigma$ range of the combined result from CMS and LHCb and for $\left|V_{c b}\right|=0.040$ one can even reach the present central experimental value of this rate. The most recent result from ATLAS [56], while not accurate, appears to confirm this picture. On the other hand the maximal shifts in the Wilson coefficient $C_{9}$ are $C_{9}^{\mathrm{NP}}=-0.1$ and $C_{9}^{\mathrm{NP}}=-0.2$ for these two $\left|V_{c b}\right|$ values, respectively. This is only a moderate shift and these models do not really help in the case of $B_{d} \rightarrow K^{*} \mu^{+} \mu^{-}$anomalies that require shifts as high as $C_{9}^{\mathrm{NP}}=-1.0[57,58]$.

- In M16 the situation is opposite. The rate for $B_{s} \rightarrow \mu^{+} \mu^{-}$can be reduced for $M_{Z^{\prime}}=3 \mathrm{TeV}$ for the two $\left|V_{c b}\right|$ values by at most $3 \%$ and $10 \%$, respectively but with the corresponding values $C_{9}^{\mathrm{NP}}=-0.3$ and -0.5 the anomaly in $B_{d} \rightarrow K^{*} \mu^{+} \mu^{-}$can be significantly reduced.

- The maximal shifts in $\varepsilon^{\prime} / \varepsilon$ decrease fast with increasing $M_{Z^{\prime}}$ in the case of $\left|V_{c b}\right|=0.042$ but are practically unchanged for $M_{Z^{\prime}}=10 \mathrm{TeV}$ when $\left|V_{c b}\right|=0.040$ is used.

- On the other hand for higher values of $M_{Z^{\prime}}$ the effects in $B_{s} \rightarrow \mu^{+} \mu^{-}$and $B_{d} \rightarrow K^{*} \mu^{+} \mu^{-}$are much smaller. NP effects in rare $K$ decays and $B \rightarrow K\left(K^{*}\right) v \bar{v}$ remain small in all 331 models even for $M_{Z^{\prime}}$ of a few TeV. This could be challenged by NA62, KOTO and Belle II experiments in this decade.

All these results are valid for $\left|V_{u b}\right|=0.0036$. For its inclusive value of $\left|V_{u b}\right|=0.0042$, we find that for $\left|V_{c b}\right|=0.040$ the maximal shifts in $\varepsilon^{\prime} / \varepsilon$ are increased to $7.7 \times 10^{-4}$ and $8.8 \times 10^{-4}$ for $M_{Z^{\prime}}=3 \mathrm{TeV}$ and $M_{Z^{\prime}}=10 \mathrm{TeV}$, respectively. Renormalization group effects are responsible for this enhancement of $\varepsilon^{\prime} / \varepsilon$ for increased $M_{Z^{\prime}}$. A recent analysis in the MSSM in [44] identifies this effect as well. But as explained in [41] eventually for very high $M_{Z^{\prime}}$, NP effects in $\varepsilon^{\prime} / \varepsilon$ will be suppressed.

Thus the main message from $[41,42]$ is that NP contributions in 331 models can simultaneously solve $\Delta F=2$ tensions, enhance $\varepsilon^{\prime} / \varepsilon$ and suppress either the rate for $B_{s} \rightarrow \mu^{+} \mu^{-}$or $C_{9}$ Wilson 
coefficient without any significant NP effects on $K^{+} \rightarrow \pi^{+} v \bar{v}$ and $K_{L} \rightarrow \pi^{0} v \bar{v}$ and $b \rightarrow s v \bar{v}$ transitions. While sizable NP effects in $\Delta F=2$ observables and $\varepsilon^{\prime} / \varepsilon$ can persist for $M_{Z^{\prime}}$ outside the reach of the LHC, such effects in $B_{s} \rightarrow \mu^{+} \mu^{-}$will only be detectable provided $Z^{\prime}$ will be discovered soon.

In this context, following our recent analysis of VLQ models (see below), let us make the following observation. Determining one day the amount of NP contributions to $\Delta F=2$ and $\Delta F=1$ processes necessary to explain the data, one will be able in a given 331 model to determine $M_{Z^{\prime}}$ necessary to explain these contributions independently of the new mixing parameters in that model. This is clearly seen in the formula (161) in [51]. It should be stressed that this is only possible by invoking both $\Delta F=2$ and $\Delta F=1$ transitions which exhibit different $M_{Z^{\prime}}$ dependence. Within $\Delta F=2$ transitions or $\Delta F=1$ transitions alone this is not possible as clearly seen in the expressions in Section 7.2 in [51].

\subsection{Models with vector-like quarks(VLQs)}

Very recently we have analysed flavour violation patterns in the $K$ and $B_{s, d}$ sectors in eleven models with VLQs [59]. I will describe here mainly the results obtained in five of them in which the gauge group is the SM one and the only new particles are VLQs in a single complex representation under the SM gauge group. A general classification of such models and references to the rich literature can be found in [60]. In these models $\Delta F=1 \mathrm{FCNCs}$ are dominated by tree-level $Z$ exchanges, while $\Delta F=2$ transitions by box diagrams with VLQs and scalars provided $M_{\mathrm{VLQ}} \geq 5 \mathrm{TeV}$. Otherwise tree-level $Z$ contributions cannot be neglected.

The summary of patterns of flavour violation in these models can be found in three DNA tables (Tables 5, 6, 10 in [59]) and the numerical results in Tables 8 and 9 in that paper. Our extensive numerical analysis has shown that NP effects in several of these models can be still very large and that simultaneous consideration of several flavour observables should allow to distinguish between these models. In particular models with left-handed and right-handed flavour violating currents can be distinguished from each other in this manner. Here we summarize the highlights of this paper.

- All tensions between $\Delta M_{s, d}$ and $\varepsilon_{K}$ can be easily removed in these models because the usual CMFV correlations between $\Delta M_{s, d}$ and $\varepsilon_{K}$ are not valid in them. The box diagrams with VLQs and Higgs scalar exchanges are dominantly responsible for it.

- Tree-level $Z$ contributions to $\varepsilon^{\prime} / \varepsilon$ can be large so that significant upward shift in $\varepsilon^{\prime} / \varepsilon$ can easily be obtained bringing the theory to agree with data.

- Simultaneously the branching ratio for $K^{+} \rightarrow \pi^{+} v \bar{v}$ can be significantly enhanced over its SM prediction, but only in models with flavour violating RH currents. In models with only LH currents $K^{+} \rightarrow \pi^{+} v \bar{v}$ branching ratio can have at most its SM value because of the $K_{L} \rightarrow \mu \bar{\mu}$ constraint. On the other hand the positive shift in $\varepsilon^{\prime} / \varepsilon$ implies uniquely suppression of the $K_{L} \rightarrow \pi^{0} v \bar{v}$ branching ratio with the suppression being smaller in models with RH currents. The fact that in models with RH currents $K^{+} \rightarrow \pi^{+} v \bar{v}$ can be enhanced, while $K_{L} \rightarrow \pi^{0} v \bar{v}$ suppressed is a clear signal of nonMFV sources at work. But also in models with LH currents only the correlations between the branching ratios of these two decays differ from the MFV one.

- These features distinguish VLQ-models from 331 models, discussed above, in which NP effects are dominated by $Z^{\prime}$ exchanges with the maximal shift in $\varepsilon^{\prime} / \varepsilon$ amounting to $0.8 \times 10^{-3}$ and NP effects in rare $K$ decays being very small.

- Significant suppressions of the branching ratio for $B_{s} \rightarrow \mu^{+} \mu^{-}$, in particular in models with LH currents are possible. While such effects are also possible in 331 models, they cannot be as large as in VLQ models. 
- On the other hand while 331 models can provide solutions to some LHCb anomalies, this is not possible in the VLQs models with SM gauge group and future confirmation of these anomalies could turn out to be a problem for the latter models.

Having the latter possibility in mind we have considered also two VLQ models with a heavy $Z^{\prime}$ related to $\mathrm{U}(1)_{\mathrm{L}_{\mu}-\mathrm{L}_{\tau}}$ symmetry and a single heavy scalar necessary to generate the $Z^{\prime}$ mass. These models, considered already in [61], can explain LHCb anomalies by providing sufficient suppression of the coefficient $C_{9}$ but NP effects in $B_{s} \rightarrow \mu^{+} \mu^{-}$and $K_{L} \rightarrow \mu^{+} \mu^{-}$are absent because of the absence of tree-level $Z$ FCNCs and vector diagonal $Z^{\prime}$ couplings to charged leptons. NP effects in $b \rightarrow s v \bar{v}$ transitions are small and the ones in $K^{+} \rightarrow \pi^{+} v \bar{v}$ and $K_{L} \rightarrow \pi^{0} v \bar{v}$ much smaller than in the models with the SM gauge group. Most importantly these models fail badly in explaning the $\varepsilon^{\prime} / \varepsilon$ anomaly. This is the consequence of the absence of tree-level $Z$ contributions in this model and of the Dirac structure of the loop generated $Z^{\prime}$ couplings to quarks that do not allow for NP contributions to the Wilson coefficients of $Q_{6}\left(Q_{6}^{\prime}\right)$ and $Q_{8}\left(Q_{8}^{\prime}\right)$ operators.

Adding a second Higgs doublet allows to generate tree-level $Z$ FCNCs with a different pattern of departures from the SM than in VLQ models summarized above. While NP effects in these remaining four models in $B_{s, d} \rightarrow \mu^{+} \mu^{-}$and $K_{L} \rightarrow \pi^{0} v \bar{v}$ turn out to be small, $\varepsilon^{\prime} / \varepsilon$ anomaly can be explained and $K^{+} \rightarrow \pi^{+} v \bar{v}$ can be enhanced both in the case of left-handed and right-handed couplings. Moreover, $\mathrm{NP}$ effects in $\Delta M_{K}$ can be larger than in the remaining seven models.

Future experimental results on $K^{+} \rightarrow \pi^{+} v \bar{v}, K_{L} \rightarrow \pi^{0} v \bar{v}, B_{s} \rightarrow \mu^{+} \mu^{-}$and LHCb anomalies and improved theoretical results on $\varepsilon^{\prime} / \varepsilon$ will tell us which of these VLQ models, if any, is selected by nature.

While the discovery of VLQs at the LHC would give a strong impetus to the models considered by us, non-observation of them at the LHC would not preclude their importance for flavour physics. In fact we have shown that large NP effects in flavour observables can be present for $M_{\mathrm{VLQ}}=10 \mathrm{TeV}$ and in the flavour precision era one could even be sensitive to higher masses. In this context we have pointed out that

- the combination of $\Delta F=2$ and $\Delta F=1$ observables in a given meson system allows to determine the masses of VLQs in a given representation independently of the size of Yukawa couplings.

\section{Outlook}

Our outlook is very short. The future of kaon flavour physics looks great and the coming years should be very exciting. I am looking forward to QCD@Work 2018 when the landscape of NP will be more transparent than it is now.

\section{Acknowledgements}

It is a pleasure to thank Monika Blanke, Fulvia De Fazio and Jean-Marc Gérard for very efficient studies discussed in this write-up and Christoph Bobeth, Alejandro Celis and Martin Jung for a very extensive analysis of VLQ models. I would also like to thank the organizers of this workshop for inviting me to this interesting event and for an impressive hospitality. This research was done and financed in the context of the ERC Advanced Grant project "FLAVOUR"(267104). It was also partially supported by the DFG cluster of excellence "Origin and Structure of the Universe".

\section{References}

[1] A.J. Buras, PoS EPS-HEP2015, 602 (2015), 1510.00128. 
[2] A.J. Buras, The Renaissance of Kaon Flavour Physics (2016), 1606.06735.

[3] A.J. Buras, PoS FWNP, 003 (2015), 1505.00618.

[4] T. Blum et al., Phys. Rev. D91, 074502 (2015), 1502 .00263.

[5] Z. Bai et al. (RBC, UKQCD), Phys. Rev. Lett. 115, 212001 (2015), 1505.07863.

[6] A.J. Buras, M. Gorbahn, S. Jäger, M. Jamin, JHEP 11, 202 (2015), 1507.06345.

[7] J. Batley et al. (NA48), Phys. Lett. B544, 97 (2002), hep-ex/0208009.

[8] A. Alavi-Harati et al. (KTeV), Phys. Rev. D67, 012005 (2003), hep-ex/0208007.

[9] E. Abouzaid et al. (KTeV), Phys. Rev. D83, 092001 (2011), 1011.0127.

[10] T. Kitahara, U. Nierste, P. Tremper (2016), 1607.06727.

[11] A.J. Buras, M. Jamin, M.E. Lautenbacher, P.H. Weisz, Nucl. Phys. B370, 69 (1992).

[12] A.J. Buras, M. Jamin, M.E. Lautenbacher, P.H. Weisz, Nucl. Phys. B400, 37 (1993), hep-ph/9211304.

[13] A.J. Buras, M. Jamin, M.E. Lautenbacher, Nucl. Phys. B400, 75 (1993), hep-ph/9211321.

[14] M. Ciuchini, E. Franco, G. Martinelli, L. Reina, Phys. Lett. B301, 263 (1993), hep-ph/9212203.

[15] A.J. Buras, M. Jamin, M.E. Lautenbacher, Nucl. Phys. B408, 209 (1993), hep-ph/9303284.

[16] M. Ciuchini, E. Franco, G. Martinelli, L. Reina, Nucl. Phys. B415, 403 (1994), hep-ph/9304257.

[17] A.J. Buras, P. Gambino, U.A. Haisch, Nucl. Phys. B570, 117 (2000), hep-ph/9911250.

[18] M. Gorbahn, U. Haisch, Nucl. Phys. B713, 291 (2005), hep-ph/0411071.

[19] J. Brod, M. Gorbahn, Phys. Rev. D82, 094026 (2010), 1007.0684.

[20] A.J. Buras, J.M. Gerard, JHEP 12, 008 (2015), 1507.06326.

[21] A.J. Buras, J.M. Gérard, Nucl. Phys. B264, 371 (1986).

[22] W.A. Bardeen, A.J. Buras, J.M. Gérard, Phys. Lett. B180, 133 (1986).

[23] A.J. Buras, J.M. Gérard, Phys. Lett. B192, 156 (1987).

[24] A.J. Buras, D. Buttazzo, J. Girrbach-Noe, R. Knegjens, JHEP 11, 033 (2015), 1503.02693.

[25] A.J. Buras, J.M. Gerard (2016), 1603.05686.

[26] V. Antonelli, S. Bertolini, M. Fabbrichesi, E.I. Lashin, Nucl. Phys. B469, 181 (1996), hep-ph/9511341.

[27] S. Bertolini, J.O. Eeg, M. Fabbrichesi, Nucl. Phys. B476, 225 (1996), hep-ph/9512356.

[28] E. Pallante, A. Pich, Phys. Rev. Lett. 84, 2568 (2000), hep-ph/9911233.

[29] E. Pallante, A. Pich, Nucl. Phys. B592, 294 (2001), hep-ph/0007208.

[30] M. Buchler, G. Colangelo, J. Kambor, F. Orellana, Phys. Lett. B521, 29 (2001), hep-ph/0102289.

[31] M. Buchler, G. Colangelo, J. Kambor, F. Orellana, Phys. Lett. B521, 22 (2001), hep-ph/0102287.

[32] E. Pallante, A. Pich, I. Scimemi, Nucl. Phys. B617, 441 (2001), hep-ph/0105011.

[33] M. Blanke, A.J. Buras, Eur. Phys. J. C76, 197 (2016), 1602.04020.

[34] A. Bazavov et al. (Fermilab Lattice, MILC), Phys. Rev. D93, 113016 (2016), 1602.03560.

[35] A.J. Buras, D. Buttazzo, J. Girrbach-Noe, R. Knegjens, JHEP 1411, 121 (2014), 1408.0728.

[36] A.J. Buras, D. Buttazzo, R. Knegjens, JHEP 11, 166 (2015), 1507.08672.

[37] A.J. Buras, JHEP 04, 071 (2016), 1601.00005.

[38] A.J. Buras, F. De Fazio, J. Girrbach, JHEP 1302, 116 (2013), 1211 . 1896.

[39] Y. Grossman, Y. Nir, Phys. Lett. B398, 163 (1997), hep-ph/9701313.

[40] M. Blanke, A.J. Buras, S. Recksiegel, Eur. Phys. J. C76, 182 (2016), 1507.06316. 
[41] A.J. Buras, F. De Fazio, JHEP 03, 010 (2016), 1512.02869.

[42] A.J. Buras, F. De Fazio, JHEP 08, 115 (2016), 1604.02344.

[43] M. Tanimoto, K. Yamamoto (2016), 1603.07960.

[44] T. Kitahara, U. Nierste, P. Tremper (2016), 1604.07400.

[45] M. Endo, S. Mishima, D. Ueda, K. Yamamoto (2016), 1608.01444.

[46] M. Singer, J.W.F. Valle, J. Schechter, Phys. Rev. D22, 738 (1980).

[47] F. Pisano, V. Pleitez, Phys. Rev. D46, 410 (1992), hep-ph/9206242.

[48] P.H. Frampton, Phys. Rev. Lett. 69, 2889 (1992).

[49] R. Foot, O.F. Hernandez, F. Pisano, V. Pleitez, Phys. Rev. D47, 4158 (1993), hep-ph/9207264.

[50] J.C. Montero, F. Pisano, V. Pleitez, Phys. Rev. D47, 2918 (1993), hep-ph/9212271.

[51] A.J. Buras, F. De Fazio, J. Girrbach, M.V. Carlucci, JHEP 1302, 023 (2013), 1211.1237.

[52] A.J. Buras, F. De Fazio, J. Girrbach, JHEP 1402, 112 (2014), 1311.6729.

[53] A.J. Buras, F. De Fazio, J. Girrbach-Noe, JHEP 1408, 039 (2014), 1405.3850.

[54] R.A. Diaz, R. Martinez, F. Ochoa, Phys. Rev. D72, 035018 (2005), hep-ph/0411263.

[55] A. Carcamo Hernandez, R. Martinez, F. Ochoa, Phys. Rev. D73, 035007 (2006), hep-ph/0510421.

[56] M. Aaboud et al. (ATLAS) (2016), 1604.04263.

[57] W. Altmannshofer, D.M. Straub, Eur. Phys. J. C75, 382 (2015), 1411. 3161.

[58] S. Descotes-Genon, L. Hofer, J. Matias, J. Virto (2015), 1510.04239.

[59] C. Bobeth, A.J. Buras, A. Celis, M. Jung (2016), 1609.04783.

[60] K. Ishiwata, Z. Ligeti, M.B. Wise, JHEP 10, 027 (2015), 1506.03484.

[61] W. Altmannshofer, S. Gori, M. Pospelov, I. Yavin, Phys. Rev. D89, 095033 (2014), 1403.1269. 